\title{
Equilibrium-Limited Periodic Separating Reactors
}

\begin{abstract}
A new unit operation is presented that utilizes a rapid feed pressure swing cycle in a bed packed with catalyst and adsorbent to effect both reaction and separation. This hybrid device combines features of a cyclic-steady-state pressure swing adsorber with those of a flowforced catalytic reactor. Feed sequences for the periodic separating reactor (PSR) are those of rapid, single-bed pressure swing adsorbers (PSA). Only the case of extremely fast reactions is considered here. A perturbed reaction-sorption equilibrium model is formulated and solved for isothermal operation for different equilibrium constants and reaction stoichiometries. The capacity and separation performance for an equilibrium-limited PSR (EPSR) can be of the same order of magnitude as PSA alone. For reactions involving a single reactant or single product, the principal component in a particular exit stream depends upon both the reaction stoichiometry and feed fraction of the process cycle. The pressure dependency of the reaction equilibrium expression is the cause of separation reversals as parameters are varied.
\end{abstract}

\author{
Garo G. Vaporciyan and \\ R. H. Kadlec \\ Department of Chemical Engineering \\ University of Michigan \\ Ann Arbor, Ml 48109
}

\section{Introduction}

Many traditional unit operations have been studied under controlled cyclic operation since the principle was first reported by Cannon (1952). In addition, several unit operations have been introduced that have no steady state analogue. These pulsed operations utilize repetitive parameter changes, so that the system behavior remains transient in a cyclic steady state. Such cyclic operation may result in improved efficiencies and increases in throughput for such processes as absorption, extraction crystallization, ion exchange, reaction, distillation, adsorption, and particle separation. Recent mass production examples of cyclic reactors include catalytic converters and gas heating furnaces.

Research in cyclic chemical operations has, until recently, concentrated on either separation or reaction processes. Several approaches have been employed to achieve cyclic separations, the most common being the cycling of any thermodynamic variable which affects the separation. Temperature, concentration, electric field, $\mathrm{pH}$, and pressure have all been examined as the cyclic variable. Separation processes of this nature depend upon the cyclic variation of the distribution coefficient for a solute between phases. One phase acts as a capacitor, alternately storing and losing solute to the other phase involved. In some cyclic separation schemes, such as parametric pumping and pressure swing adsorption, the process includes a flow reversal that is coupled to a change in a thermodynamic variable.

Reactor performance is altered by cyclic operation for any nonlinear process, as discussed by Bailey (1973), Sincic and Bailey (1977), and recently Kevrekidis et al. (1986). For heterogeneous catalytic gas phase reactors, surface rate processes such as adsorption, diffusion, surface reaction, and desorption are influenced by the periodic operation, even to the point of changing the dominant reaction mechanism. Feed concentration is the variable usually cycled.

In this paper, we combine the cyclic separation process of pressure swing adsorption (PSA) with a cyclic reaction process. Although such a hybrid device could utilize any of the multibed, single-bed, or rotational forms of PSA, only the single-bed version is considered here. The objective of this study was to attain an understanding of the behavior and mechanism of the periodic process for various reaction stoichiometries within a limited operating region: isothermal, equilibrium operation for constant feed composition.

The periodic separating reactor (PSR) is constructed and operated like a single-bed pressure swing adsorber, Figures 1 and 2. The column contains a combined mixture of catalyst and adsorbent. The gaseous reactants are pumped into the packed bed under high pressure for a fraction of the operating cycle. 


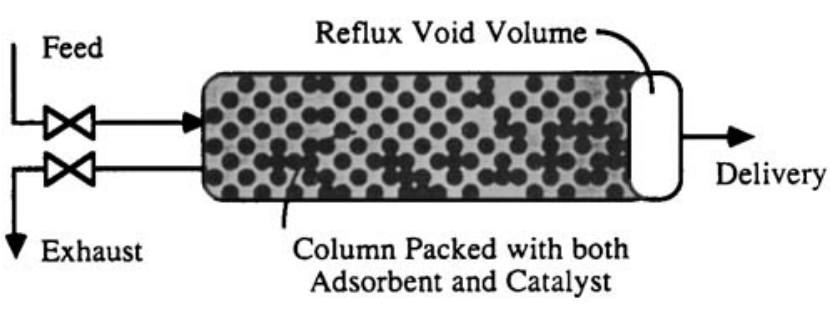

Figure 1. Periodic separating reactor.

The feed end may be dead-ended for a short time before being allowed to exhaust for the duration of the cycle. Due to the low permeability of the packing, a continuous stream can be removed at the opposite end of the column. A void volume is placed between the reaction bed and the delivery stream to allow for reflux and perhaps higher product purities, as with singlebed PSA (Kowler and Kadlec, 1972). After a start-up period consisting of many cycles, a stationary (repetitive) cyclic steady state is reached, with periodically varying pressure and composition existing in the bed. The chemical conversion and separation accomplished depend upon the manner in which the pressure and reactant concentrations are controlled at the feed boundary.

It is significant that this process has no steady state analogue and therefore cannot be described against the background of steady state theory. The separation and capacity performance of an equilibrium-limited PSR (EPSR) is of the same order of magnitude as that of equilibrium PSA alone. The component enriched in a particular EPSR exit stream has been found to depend upon both the reaction stoichiometry and the feed fraction of the process cycle. The perturbed reaction-sorption model also predicts that a shorter length periodic reactor can process more reactants without a loss of conversion or separation. PSR's may provide a recycle stream without phase change or extractive procedures while reducing catalytic requirements for production units. Joined with the multibed and rotational versions, a whole new spectrum of processes emerges for potential use by industry.

\section{Pressure Effects on Equilibrium}

For a single gas phase reaction exhibiting ideal solution behavior, thermodynamics predicts the effects of total pressure and the net change in moles due to reaction upon the equilibrium conversion obtainable. Equilibrium conversion increases

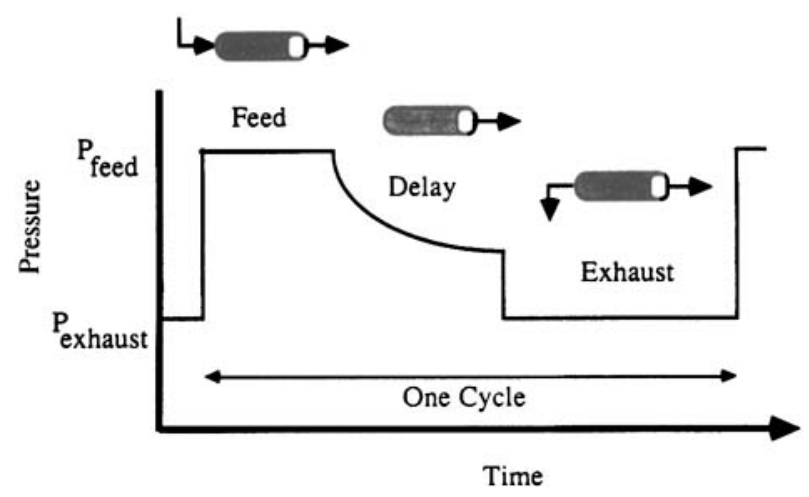

Figure 2. Characteristics of inlet pressure wave form. with increasing reactant partial pressures for reactions that have a net decrease in the number of moles. Reactions that have a net increase in the number of moles exhibit increasing equilibrium conversion with decreasing reactant partial pressures. Pressure transients thus cause reaction to occur, as illustrated by the following simple example.

Consider a solid catalyzed gas phase reaction at equilibrium in a thermostated closed canister, according to the chemical equation: $A+2 B \rightarrow A B_{2}$, and at the conditions given in Table 1 . A valve is opened, and the contents are depressurized. This outflow is slow enough that the contents remain isothermal, and both chemical and sorption equilibria are maintained at all times. A linear adsorption isotherm was employed. The model equations were transformed by replacing time with the total molar output, $O$, which is related to real time by the equation $d O=$ (molar outflow) $d t$. The change in the exit mole fractions of each component with $O$ are presented in Figure 3. The mole fraction of the product $A B_{2}$ drops tenfold during the tank depressurization. A series of such interconnected tanks, with controlled terminal flows, represents a discretized version of a periodic separating reactor.

\section{The Perturbed Equilibrium Model}

Modeling of periodically operated heterogeneous catalytic reactors has focused on rate improvements on the basis of adsorption-desorption phenomena. Open arguments exist in the literature concerning the ability of such adsorption-desorption models to predict rate improvements and product selectivities due to periodic operation (Lynch, 1984; Amariglio and Rambeau, 1984; Jain et al., 1983; Feimer et al., 1982). The difficulty of modeling cyclic reactors, even qualitatively, is well known (Mihail and Paul, 1979; Jain et al., 1981; Imbihl et al., 1985; Salmi et al., 1986). Rate models developed from steady-state experiments do not hold for cyclic states. The steps in a reaction mechanism that are rate limiting may depend upon the manner in which the reactor is operated (Cutlip, 1979; Lynch et al., 1986). Researchers are investigating island theories and formulating stochastic and Monte Carlo simulations in order to account for the interactions of species on and with the catalyst surface (Williams et al., 1982; Lynch et al., 1986).

On the other hand, the performance of single-bed, rapid pressure swing adsorbers has been successfully modeled and optimized for two-component separations (Turnock and Kadlec, 1971; Kowler and Kadlec, 1972).

Within a periodic separating reactor, even when adsorption and desorption rate phenomena are considered at local equilibrium, heterogeneous surface concentrations are not constant with

Table 1. Single-Tank Depressurization Parameters

\begin{tabular}{|c|c|c|c|}
\hline Parameter & & Value & \\
\hline \multirow[t]{2}{*}{$\begin{array}{l}\text { Initial tank press., atm } \\
\text { Temp., K } \\
K_{\text {eq }}, \text { atm }^{-2}\end{array}$} & & $\begin{array}{c}3.0 \\
298 \\
0.50\end{array}$ & \\
\hline & $A$ & $\begin{array}{c}\text { Componen } \\
B\end{array}$ & $A B_{2}$ \\
\hline $\begin{array}{l}\text { Apparent vol. } V \gamma_{i}, \mathrm{~cm}^{3} \\
\text { Initial mol frac., at equilibrium }\end{array}$ & $\begin{array}{l}134 \\
0.4437\end{array}$ & $\begin{array}{l}73.7 \\
0.3333\end{array}$ & $\begin{array}{l}13.5 \\
0.2219\end{array}$ \\
\hline
\end{tabular}




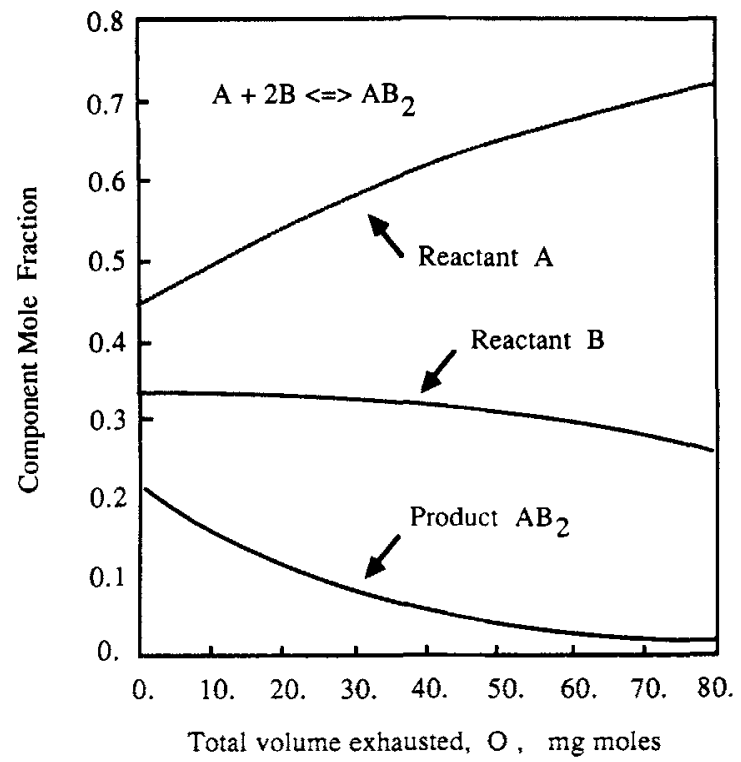

Figure 3. Mole fractlons vs. total output $O$, during depressurization of a single tank.

respect to time. Due to fluctuating pressures, modeling must include accumulation terms for both gas and solid phases, even at cyclic steady state. If local reaction rates are fast enough, local reaction equilibrium may be assumed. As with sorption equilibrium, local reaction equilibrium will vary cyclically, thus the perturbed equilibrium model.

Consider a periodic separating reactor in which a reaction proceeds involving $N$ components. Mathematical model formulation involves these simplifying assumptions:

1. Local sorption equilibrium

2. Local reaction equilibrium

3. Isothermal operation

4. No radial concentration and/or pressure gradients

5. The process is not diffusion limited in any respect

6. Ideal gas behavior

7. Darcy's law for flow through packed beds

8. Linear sorption isotherms

9. Ideal solution behavior in the gas phase

10. Perfect square-wave feed-exhaust pressure

11. No variations in the feed concentration

12. Well-mixed void volume at the far end of the reactor

13. No void volume at the feed end of the packed bed

14. Constant molar delivery stream flow rate

Most of the assumptions are valid for operation of a single-bed pressure swing adsorber without reaction at sufficiently high temperature (Turnock and Kadlec, 1971). The reaction equilibrium assumption would be valid at high temperatures for highly active catalysts and thus represents one limit of the operating region for PSR's.

A general single reaction of the form $\Sigma v_{l} S_{i}=0$ is considered. The state equations may be formed from conservation laws and thermodynamics. After being made dimensionless, total mass conservation, including both gas and solid phases gives:

$$
\frac{\partial}{\partial \lambda}\left\{\left(\frac{\tau P_{j} k}{\mu L^{2}}\right) Z\left[\sum_{i=1}^{N} y_{i} M_{i}\right] \frac{\partial Z}{\partial \lambda}\right\}=\frac{\partial}{\partial \theta}\left\{Z\left[\sum_{i=1}^{N} y_{i} M_{i} \gamma_{i}\right]\right\}
$$

where the dimensionless capacity is:

$$
\gamma_{i}=\epsilon+\rho R T \alpha_{i} \quad \text { for } i=1,2, \ldots, N
$$

The local reaction equilibrium, expression is:

$$
\prod_{i=1}^{N} y_{i}^{v_{i}}=K_{e q} P_{f}^{-\Delta v} Z^{-\Delta v}
$$

where the net change in moles due to reaction is:

$$
\Delta v=\sum_{i=1}^{N} v_{l}
$$

A balanced chemical equation requires the relations:

$$
\sum_{i=1}^{N} \eta_{j, i} v_{i}=0 . \quad j=1,2, \ldots, J
$$

where $J$ is the rank of the atom matrix.

In addition, $\mathrm{J}-2$ independent atom balances (the remaining two are replaced by Eqs. 3 and 7) must be written because moles are not necessarily conserved. These atom balances are similar to the mass balance in form, and may be written as:

$$
\frac{\partial}{\partial \lambda}\left\{\left(\frac{\tau P_{f} k}{\mu L^{2}}\right) Z\left[\sum_{i=1}^{N} y_{i} \eta_{j, i}\right] \frac{\partial Z}{\partial \lambda}\right\}=\frac{\partial}{\partial \theta}\left\{Z\left[\sum_{i=1}^{N} y_{i} \eta_{j, i} \gamma_{l}\right]\right\}
$$

As always a mole fraction constraint exists:

$$
\sum_{i=1}^{N} y_{i}=1
$$

The above $J+1$ state equations, Eqs. 1-7, are the basis of the model, along with time and boundary conditions, and similar reflux void volume conservation laws. For the reflux void volume the following equations apply.

Mass conservation:

$$
\frac{\partial Z_{R}}{\partial \theta}=-\left.\left(\frac{\tau P_{f} k}{\mu L^{2}}\right)\left(\frac{A L}{V_{R}}\right) Z \frac{\partial Z}{\partial \lambda}\right|_{\lambda=1}-\left(\frac{Q_{d} R T \tau}{P_{f} V_{R}}\right)
$$

Composition, depending upon the direction of flow at the packed bed reflux void volume interface:

For flow from the packed bed into the reflux void volume, i.e.,

when

$$
\begin{aligned}
\left.\frac{\partial Z}{\partial \lambda}\right|_{\lambda-1}<0 \quad \text { then for } i & =1,2, \ldots, N \\
\qquad \frac{\partial y_{R i}}{\partial \theta} & =\left.\left(\frac{\tau P_{f} k}{\mu L^{2}}\right)\left(\frac{A L}{V_{R}}\right) \frac{Z}{Z_{R}}\left(y_{R i}-y_{i}\right) \frac{\partial Z}{\partial \lambda}\right|_{\lambda-1}
\end{aligned}
$$

During reflux, for flow from the void volume into the packed bed, i.e., 
when

$$
\left.\frac{\partial Z}{\partial \lambda}\right|_{\lambda-1} \geq 0 \quad \text { then for } i=1,2, \ldots, N \quad \frac{\partial y_{R I}}{\partial \theta}=0
$$

For any feed boundary pressure control and delivery flow rate, the system is defined. Boundary conditions for the packed catalytic bed are:

At the feed end, $\lambda=0$, during the feed portion of the cycle,

$$
Z(0, \theta)=1 ., \quad y_{i}(0, \theta)=y_{f, i} \quad \text { for } i=1,2, \ldots, N
$$

during the delay portion of the cycle,

$$
\left.\frac{\partial Z}{\partial \lambda}\right|_{\lambda=0}=0
$$

and during the exhaust portion of the cycle $Z(0, \theta)=Z_{\min }$.

At the delivery end, $\lambda=1$,

when

$$
\begin{aligned}
& \left.\frac{\partial Z}{\partial \lambda}\right|_{\lambda=1}>0 \text { then, } \quad Z(1, \theta)=Z_{R}(\theta) \\
& \qquad y_{i}(1, \theta)=y_{R, i}(\theta) \quad \text { for } i=1,2, \ldots, N
\end{aligned}
$$

The above set of equations describes transients in equilibrium limited periodic separating reactors. Cyclic steady state is achieved when the conditions within the packed bed and the reflux volume repeat every cycle. The perdiocity conditions are:

$$
\begin{aligned}
Z(\lambda, \theta)=Z(\lambda, \theta+1), \quad y_{i}(\lambda, \theta)=y_{i}(\lambda, \theta+1) \\
\\
\text { for } i=1,2, \ldots, N
\end{aligned}
$$

$Z_{R}(\theta)=Z_{R}(\theta+1), \quad y_{R, i}(\theta)=y_{R, i}(\theta+1)$

$$
\text { for } i=1,2, \ldots, N
$$

The total dimensional exhaust rate is calculated from:

$$
Q_{e}=\left.\left(\frac{A L P_{f}}{\tau R T}\right)\left(\frac{\tau P_{f} k}{\mu L^{2}}\right) Z_{\min } \int_{f_{f}+f_{D}}^{1} \frac{\partial Z}{\partial \lambda}\right|_{\lambda=0} d \theta
$$

The resulting perturbed equilibrium model contains $J-1$ second-order, nonlinear, coupled partial differential equations, along with two algebraic equations. The boundary conditions are time and state dependent, and include ordinary differential equations. In general, substitution can reduce the system by the mole fraction constraint, Eq. 7 . The resulting equations that must be solved may be termed a coupled, second-order, parabolic, nonlinear partial algebraic equation (PAE) set.

Examination of the model reveals four classes of quantities:

Independent Variables. Dimensionless length down the reactor, $\lambda$, ( 0 at the feed end, 1 at the reflux void volume end), dimensionless cycle time, $\theta$, $(0$ at the start of a cycle, and 1 at the end).

Design Parameters. Feed pressure, exhaust pressure, delivery flow rate, cycle time, feed fraction of the process period, delay fraction of the process period, feed composition, reflux void vol- ume, length of the reactor, temperature, cross-sectional area, and the type of catalyst and adsorbent employed.

Physical Parameters. Dimensionless groups and constants for linear adsorption isotherms and Darcy's law, reaction equilibrium constant, reaction atom stoichiometry, molecular weights, packing void volume, and viscosity.

Dependent Variables. Dimensionless pressure $Z$, and $Z_{R}$, along with the mole fractions of each component in both the PSR and reflux void volume.

These form into nine dimensionless parameters that characterize the device.

\section{Perturbed Equilibrium Simulator}

An algorithm for solving the perturbed equilibrium model for any reaction involving three components was written. The solution of two second-order, parabolic, nonlinear PDE's, Eqs. 1 and 6 , and a nonlinear algebraic equation, Eq. 3, became necessary. An implicit finite-difference method, with a Newton-Raphson scheme for the solution of the nonlinear difference equations, was attempted. The computer program successfully simulated pressure swing adsorption alone. A nontrace two-component system that has previously been studied (the methane-nitrogen system investigated by Turnock and Kadlec, 1971, and Kowler and Kadlec, 1972) served as the test case. System performance predicted by the simulator was in better agreement with the actual experimental data than that in earlier work.

The addition of the reaction equilibrium algebraic constraint resulted in unstable behavior. Although the numerical methods employed are recommended for differential algebraic equation (DAE) systems, performance on the PAE system at hand was poor. Nonlinear instabilities existed, of the type described by Adam (1985), which are common for even the simplest transport equations with periodic boundary conditions. Spurious oscillations can occur (even in linear systems) because numerical schemes propagate the elementary waves forming the solution at different speeds. For these reasons, a cell discretization was implemented using a finite-difference scheme.

Solution of the resulting coupled nonlinear DAE system was accomplished without the numerical problems that appeared earlier. The algorithm was designed to be efficient and employed a modified Runge-Kutta-Fehlberg ODE integrator with step-size control. The majority of steps during a cycle were taken immediately after a feed boundary pressure change (from $P_{f}$ to $P_{e}$ at $\tau=0$, and from $P_{e}$ to $P_{f}$ at $\tau=f_{f}+f_{D}$

Simulation of PSA without reaction served as validation. Simulations with the current algorithm agreed with previous Eulerian and Lagrangian algorithms, which agreed with previous experimental data. Atom balances and dimensional analysis similarity provided additional checks during reaction simulations. It was determined that exhaust and delivery stream flow rates are invariant to the number of cells employed in the discretization, once above five. In general, six cells were employed for the results presented here. Exhaust and delivery stream compositions were found to be within $1 \%$ of the extrapolated infinite cell solution. Cyclic steady state was assumed when all state variables varied less than $10^{-4} \%$ from one cycle to the next. Normally this required the integration of 80-100 cycles from an initial state. Exhaust stream composition converged upon cyclic steady state prior to the delivery stream composition. On both Amdahl $470 \mathrm{~V} / 8$ and Apollo 460 computers, run times to reach cyclic steady state were on the order of $3 \mathrm{cpu}$ min and $3 \mathrm{cpu} \mathrm{h}$, 
respectively. For the EPSR systems investigated, initial conditions did not effect the cyclic-steady-state solution achieved. In addition, perturbations from a cyclic steady state resulted in dynamics that returned the EPSR to the original cyclic steady state.

\section{Simulation Results and Discussion}

Simulation studies covered several topics and included both physical and design parameters. Physical parameters investigated included reaction stoichiometry, adsorbent coefficients, and the reaction equilibrium constant. Design variables included delivery flow rate, cycle period, and feed fraction of the process period. In general, a small delivery flow rate was employed, because this was presumed to provide maximum concentration effects. Preliminary runs led to the base case operating conditions shown in Table 2. These parameter values are employed in all runs unless otherwise stated. Results were evaluated based upon degree of separation, system throughput, dimensionless flow rates, and relative conversion.

The separation factor is the ratio of mole fractions between the delivery and exhaust streams. Thus, for ratios above one, that component is enriched in the delivery stream.

The system throughput is the cycle average flow rate ( $\mathrm{mg} \cdot \mathrm{mol} / \mathrm{s}$ ) processed by the separating reactor. The cycle average dimensionless flow rate is the average flow rate for a stream during one cycle divided by the delivery flow rate.

The relative conversion is the actual conversion achieved in the PSR divided by the most favorable batch equilibrium conversion possible. Therefore, for reactions that produce moles, the relative conversion is related to the batch conversion at the lowest system pressure, while for those reactions that destroy moles, it is related to the batch equilibrium conversion at the highest system pressure. The perturbed equilibrium model must produce conversions between those attainable at the feed and

Table 2. Base Case Values for Dimensionless Groups and Varied Parameters

\begin{tabular}{|c|c|c|c|c|c|c|}
\hline & \multicolumn{6}{|c|}{ Dimensionless Group } \\
\hline & $\frac{\tau P_{f} k}{\mu L^{2}}$ & $\frac{Q_{d} R T \tau}{P_{f} V_{R}} \quad \frac{A L}{V_{R}}$ & $Z_{\min }$ & $\epsilon$ & $f_{f}$ & $f_{D}$ \\
\hline \multirow[t]{3}{*}{ Base case value } & 3.747 & $1.658 \quad 13.1$ & $11 / 3$ & $3 \quad 0.623$ & 0.50 & 0.00 \\
\hline & \multicolumn{6}{|c|}{ Dimensionless Group } \\
\hline & $K_{e q} P_{f}^{-\Delta v}$ & Component & $y_{f i t} \epsilon$ & $\epsilon+\rho R T \alpha i$ & \multicolumn{2}{|c|}{$\begin{array}{c}\eta_{j, i} / \eta_{j, A} \\
j=1\end{array}$} \\
\hline$A=2 C$ & 1.00 & $\stackrel{A}{C}$ & $\begin{array}{l}1.0 \\
0.0\end{array}$ & $\begin{array}{l}6.25 \\
0.62\end{array}$ & \multicolumn{2}{|c|}{$\begin{array}{l}1.0 \\
0.5\end{array}$} \\
\hline $2 A-C$ & 9.00 & $\begin{array}{l}\boldsymbol{A} \\
\boldsymbol{C}\end{array}$ & $\begin{array}{l}1.0 \\
0.0\end{array}$ & $\begin{array}{l}6.25 \\
0.62\end{array}$ & \multicolumn{2}{|c|}{$\begin{array}{r}1.0 \\
2.0 \\
j=1 \quad j=2 \\
\end{array}$} \\
\hline \multirow[t]{3}{*}{$A-1 / 2 B+C$} & 1.73 & $\begin{array}{l}A \\
B \\
C\end{array}$ & $\begin{array}{l}1.0 \\
0.0 \\
0.0\end{array}$ & $\begin{array}{l}6.25 \\
0.62 \\
6.25\end{array}$ & $\begin{array}{l}1.0 \\
0.0 \\
1.0\end{array}$ & $\begin{array}{l}1.0 \\
1.0 \\
0.5\end{array}$ \\
\hline & & \multicolumn{3}{|c|}{ Parameter } & & \\
\hline & & $\begin{array}{c}Q_{d} \\
\mathrm{mg} \mathrm{mol} / \mathrm{s}\end{array}$ & $\operatorname{atm}^{K_{\text {eq }}}$ & $\begin{array}{l}\tau \\
\text { s }\end{array}$ & & \\
\hline Base case value & & 1.6286 & 3.0 & 5.0 & & \\
\hline
\end{tabular}

exhaust pressures. Relative conversions thus never exceed $100 \%$ in a model that allows for instantaneous reaction equilibrium between reactant and product. As will become evident, a loss in conversion is exchanged for an enhancement in separation.

\section{Physical Parameters}

The simple system $v_{A} A \rightarrow v_{C} C$, employed without a diluent, provides three different cases: moles being created, destroyed, or conserved. Where total moles are conserved, the reaction equilibrium expression, Eq. 2, is independent of pressure, i.e., $\Delta v=$ 0 . Thus, the perturbed equilibrium model predicts no separation or improvement in conversion. The delivery and exhaust streams have the same composition, that of the reactant mixture converted to equilibrium.

The effect of moles being created or destroyed by the reaction upon the separation and conversion is plotted in Figure 4. Although the reactant is preferentially adsorbed, the component (reactant or product) that is enriched in the delivery stream depends upon whether the reaction creates or destroys moles. The enrichment becomes more pronounced with an increase in the net change in moles due to reaction. This result is the direct consequence of the pressure dependence of the reaction equilibrium expression. Pressure swing adsorption theory alone would predict reactant enrichment in the exhaust stream under all conditions.

Physical adsorption properties may not exert the same effect in EPSR as in PSA. For the two-component periodic reactor, a reversal in the adsorptive capacities of the bed does not cause a separation reversal as it would in a PSA process. For a reaction that has more than one product, such as $A-1 / 2 B+C$, the adsorptive properties exert an effect similar to PSA for the products (e.g., if the adsorbent preferentially adsorbs component $B$ over $C$ than $B$ will be enriched in the exhaust stream.) Separation of the unconverted reactant is poor regardless of adsorbent selectivity.

The adsorptive capacities do however influence the throughput of the separating reactor. The larger the total affinity for the components present, the larger the throughput of the periodic reactor. For a fixed total molar adsorption isotherm, increase in

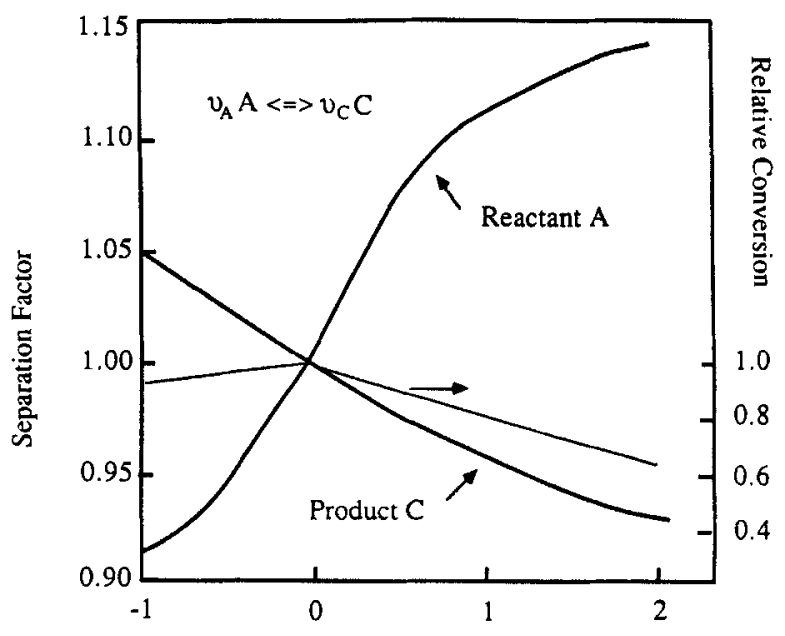

Net Change in Moles due to Reaction, $\Delta v$

Figure 4. Net change in moles due to reaction vs. separation factor and relative conversion. 
the selectivity of the product(s) leads to an increase in process throughput. For example, in the case of $A-2 B$, reversal of the adsorption affinity from reactant-only to product-only, doubles the $\mathrm{mol} / \mathrm{s}$ processed by the reacting separator. Actual and relative conversions of the feed are only slightly affected by the adsorptive capacities, increasing $1-2 \%$ for a $100 \%$ increase in the total or individual adsorption capacities.

Simulations were conducted varying the equilibrium constant over several orders of magnitude. Results are presented in Figure 5 for $A \rightarrow 2 C$. Actual and relative conversions increase as $K_{\text {eq }}$ is increased. The same is true for the stoichiometries $2 A-$ $C$, and $A \rightarrow 1 / 2 B+C$.

The dimensionless flow rates of an EPSR decrease with increasing $K_{e q}$ for $A-2 C$, Figure 6, and for $A-1 / 2 B+C$. For reactions that create moles the exhaust stream is larger than the feed, while for reactions that destroy moles the opposite is true. The net change in moles due to reaction does not explain the drop in dimensionless flow rate with increasing $K_{\text {eq }}$. Rather, this phenomenon is understood by noting that the adsorbent favors the reactant. At high conversions there is less reactant present and thus less to adsorb. Therefore, as $K_{e q}$ increases, the dimensionless flow rates of the PSR decrease regardless of the net change in moles due to reaction. Reversal of the adsorption selectivities causes the dimensionless flow rate $K_{\text {eq }}$ phenomenon to also reverse, i.e., EPSR dimensionless flow rates increase with increasing $K_{e q}$.

The separation performance of the EPSR system for a twocomponent reaction is shown in Figure 7. For reaction systems, $A \rightarrow 2 C$ or $2 A-C$, at low conversions (low $K_{e q}$ ) the reactant is not separated (i.e., enriched in one stream), however the product is. For large values of $\boldsymbol{K}_{\text {eq }}$ (high conversions) the reactant is separated while the product is not. The difference in behavior between the two reactions determines where the components accumulate. For the reaction that creates moles, the reactant is concentrated in the delivery stream and the product in the exhaust, while the opposite is true for reactions that destroy moles. This result might be unexpected because both systems have an adsorbent that preferentially adsorbs the reactant, and thus one would have expected the reactant to concentrate in the

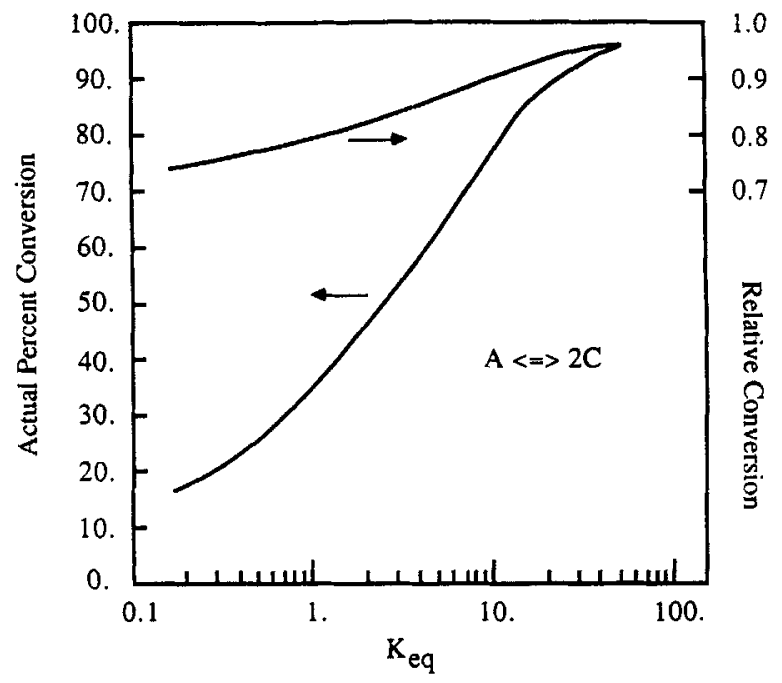

Figure 5. Reaction equillbrium constant vs. actual and relative conversion.

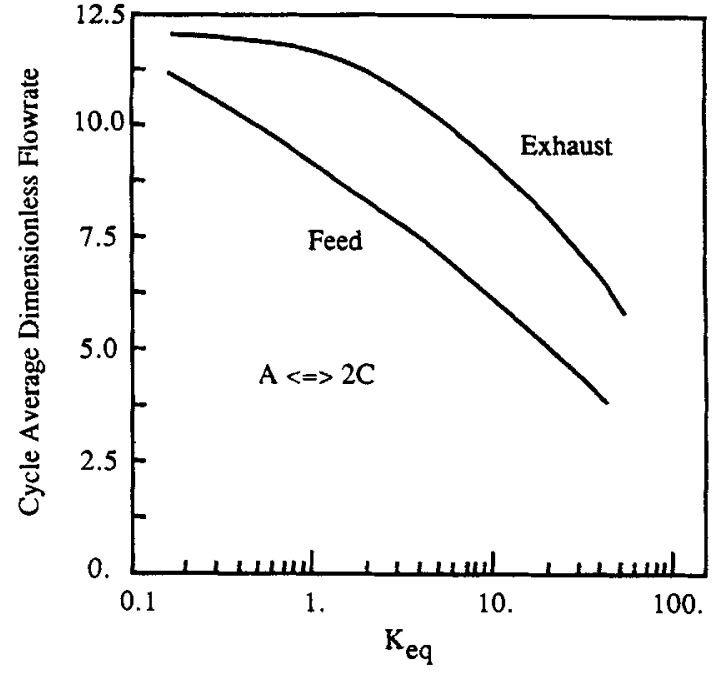

Figure 6. Reaction equilibrium constant ve. cycle average dimensionless flow rate.

exhaust stream. An explanation is found by examining the pressure dependency of the reaction equilibrium expression. During the exhaust part of the cycle, for both reaction cases, the pressure waves within the EPSR result in a higher average pressure in the delivery stream than at the feed end of the reactor. For the reaction $A-2 C$, the higher the pressure the lower the conversion of $A$ to $C$ for a fixed value of $K_{e q}$. Thus, more reactant is left at the delivery than at the exhaust end. For small values of $K_{\text {eq }}$, there is plenty of reactant and the difference between the exhaust and delivery due to the pressure effect is small in comparison. However, when the conversion is almost complete (i.e., large values of $K_{e q}$ ) the concentration difference between the exhaust and delivery due to the pressure effect is large in proportion to the total amount of reactant present.

The reverse trend for the reaction $2 A \rightarrow C$ is similarly explained.

The separation performance of the EPSR system for the reaction $A \rightarrow 1 / 2 B+C$ is quite different from that for the two-

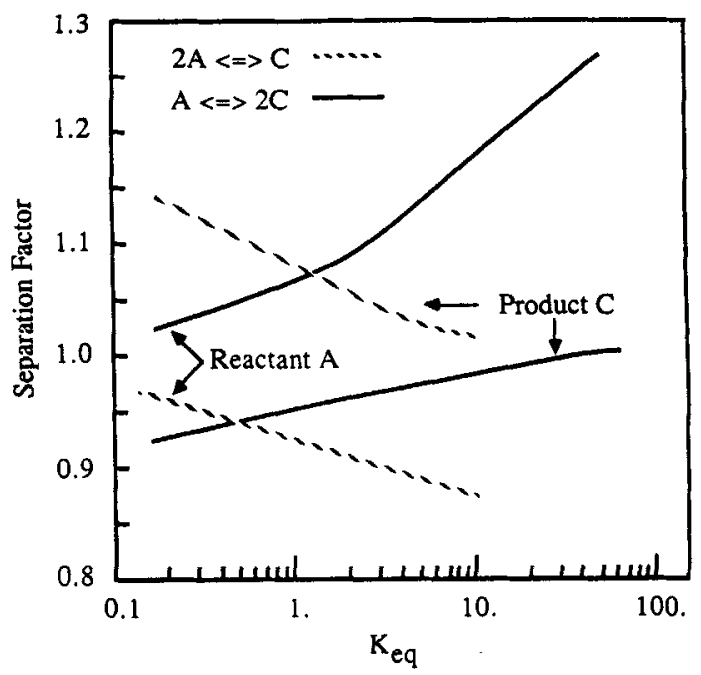

Figure 7. Reaction equilibrium constent v8. separation factor. 
component system. Separation factors for the product ( 2.0 for $B$ and 0.6 for $C$ ) are invariant over four orders of magnitude for $K_{\text {eq }}(0.1-1,000)$. The separation of the products is as expected by PSA theory. The reactant $A$ however, shows enrichment and separation $K_{e q}$ performance similar to the reactant in two-component reactions that create moles.

Separation of components that are the single reactant or product from the other components in the reactor is due only to the pressure effect on the equilibrium conversion. At a constant pressure the reaction equilibrium expression for a two-component reacting system has no degrees of freedom, thus the pressure effect is controlling. An additional product or reactant provides a degree of freedom in the reaction equilibrium constraint, thus the PSA performance is observed.

Concentration histories at different lengths into the separating reactor are given in Figure 8 for component $C$ in the reaction $A-1 / 2 B+C$. There are clearly two regions corresponding to the feed and exhaust part of the cycle. Bed pressures increase during the feed portion of the period and decrease during the exhaust portion. The product $C$ is removed from the gas phase by adsorption during the feed portion of the period and then released during the exhaust portion of the cycle. Enrichment occurs toward the feed end of the PSR. The opposite effect is observed for product $B$. Due to lack of adsorption, accumulation is in the gas phase durng the feed portion of the period and enrichment occurs toward the delivery end of the PSR.

\section{Dimensional Analysis}

A dimensional analysis of the appropriate form of the state equations shows that if the dimensionless groups,

$$
\left(\frac{Q_{d} R T \tau}{P_{f} V_{R}}\right),\left(\frac{\tau P_{f} k}{\mu L^{2}}\right),\left(\frac{A L}{V_{R}}\right),\left(K_{e q} P_{f}^{-\Delta v}\right),\left(\epsilon+\rho R T \alpha_{l}\right)
$$

and the dimensionless parameters $f_{f}, f_{D}, Z_{\min }$ and the feed mole fractions are not changed, for a particular reaction, the solution to the dimensionless state equations will not change. This means that there exist sets of parameter values that will result in the

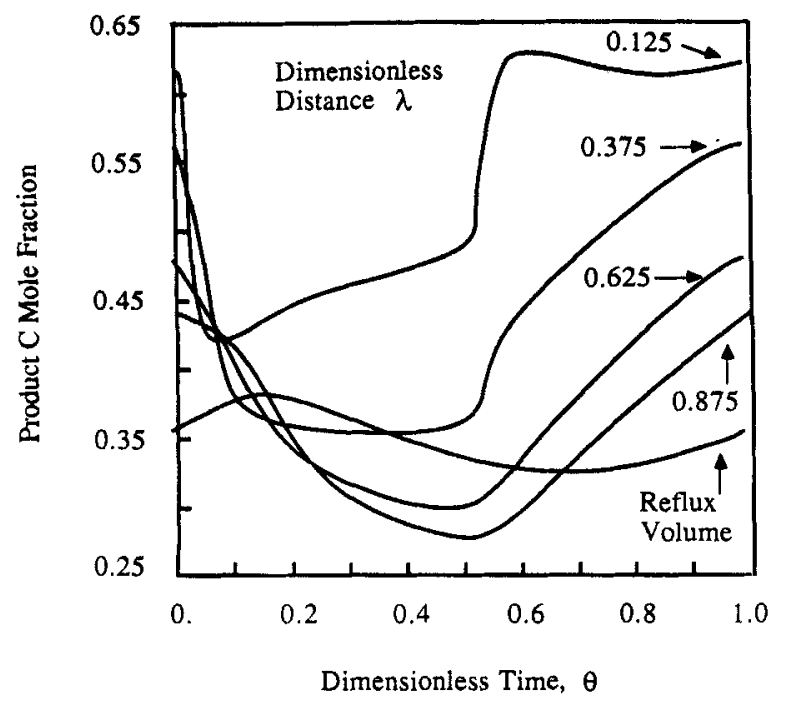

Figure 8. Concentration histories at varlous distances Into EPSR for component $C$ in reaction $A-$ $1 / 2 B+C$. same extent of separation and reaction with the EPSR. Several of the dimensionless groups identified are also common to single-bed pressure swing adsorption.

The effect of a change in various parameters may be determined through the use of the dimensionless groups above. For example, if $(k / \mu)$ is changed to $(k / \mu)^{\prime}$ the same solution will be maintained if the cycle period $\tau$ is changed by a factor of $(k / \mu) /(k / \mu)^{\prime}$ and $Q_{d}$ is changed by the inverse of this factor. For any solution of the dimensionless state equations where the above dimensionless groups are kept constant, the exhaust rate will change by the factor $\left(A L P_{f} / \tau R T\right)$.

The variation in $(k / \mu)$ that leads to a change in $\tau$ will thus change the exhaust rate by the factor $(k / \mu)^{\prime} /(k / \mu)$. Therefore, a variation in $k$ or $\mu$, if accompanied by the appropriate change in $\tau$, leads to the same separation, reaction, and fraction of the feed gas recovered in each stream. The throughput of the system, however, has been altered. The permeability and gas viscosity thus directly affect the throughput of the system but not the relationship between the fraction of feed gas recovered in the delivery stream, the separation, and the extent of reaction achieved.

Dimensional analysis may also include design parameters. As in the case of one-bed rapid PSA (at sorption equilibria), but unlike the behavior of most process equipment, a decrease in the length of the packed bed coupled with an increase in the cycling frequency will result in an increase in the throughput capacity of the system. In other words, a shorter periodic reactor can process more reactants without a loss of conversion or separation.

These results must be interpreted with caution, as faster cycles, a shorter reactor, and a larger capacity will lead to higher flow rates, perhaps requiring the introduction of rate-limiting factors that have been neglected in the formation of the equilibrium model.

\section{Design Parameters}

There exist many design parameters for the periodic separating reactor. Previous work on both PSA and concentration swing reactors has identified the importance of the input wave form. For PSA, bang-bang control has been found to be optimal. Even with this limitation there are many different process parameters that can be investigated. Within this work, only the total cycle period and the feed fraction of the period were numerically investigated via simulations. The interactions between cross sectional area, PSR length, and $\tau$ were extracted from the dimensionless groups identified earlier.

The effect of total cycle period upon the dimensionless flow rates and separation within an EPSR is shown in Figures 9 and 10. There exists a cycle period range within which the device may operate. This operating range arises due to the two limits upon the total cycle time. If the cycle time period is too short, the bed attenuation is so great that the delivery flow rate cannot be realized. At the other end of the period range, long cycle times lead to long exhaust times and thus also result in a system incapable of sustaining a set delivery flow rate. The range of operating cycle periods is therefore dependent upon the operating parameters that affect flow through the packed bed. An increase in the reflux void volume extends the range to include longer cycle periods, but does not affect the short cycle period range limit.

The effect of cycle period upon percent conversion of reactant to product(s) is slight, and no conversion enhancement is 


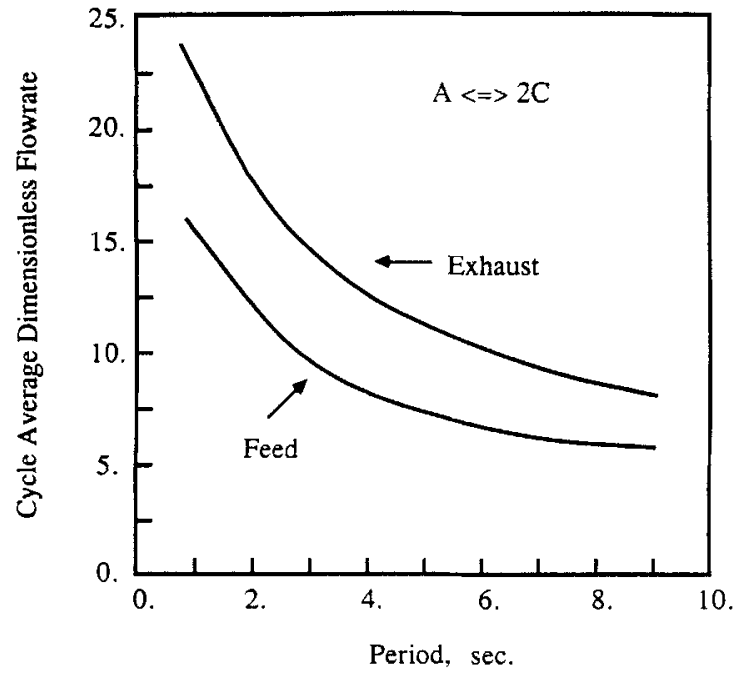

Figure 9. Period vs. cyclic average dimensionless flow rate.

achieved. For a reaction that creates moles, the conversion increases with increasing cycle period. For a reaction with a net decrease in moles, the conversion decreases with increasing cycle time. These trends may be explained by considering the pressure dependency of the reaction equilibrium expression along with the effect of the period on the average bed pressure. Rapid cycling produces bed pressures during the exhaust portion of the cycle that are higher than those produced during slow cycling. Most of the gas exits by way of the exhaust stream, thus the pressure at that end of the EPSR controls the overall conversion. Therefore, when $\Delta v$ is positive, $(A \hookrightarrow 2 C, A \hookrightarrow 1 / 2 B+C)$ the conversion increases with increasing cycle time, while for the reaction that destroys moles $(2 A-C)$ conversion decreases with increasing cycle time.

Although the total dimensionless flow rates of the EPSR decrease with increasing period, the fraction recovered in the delivery stream (delivery cut) increases, Figure 9 . For reactions that create moles the exhaust stream is larger than the total amount fed, while for reactions that destroy moles the opposite

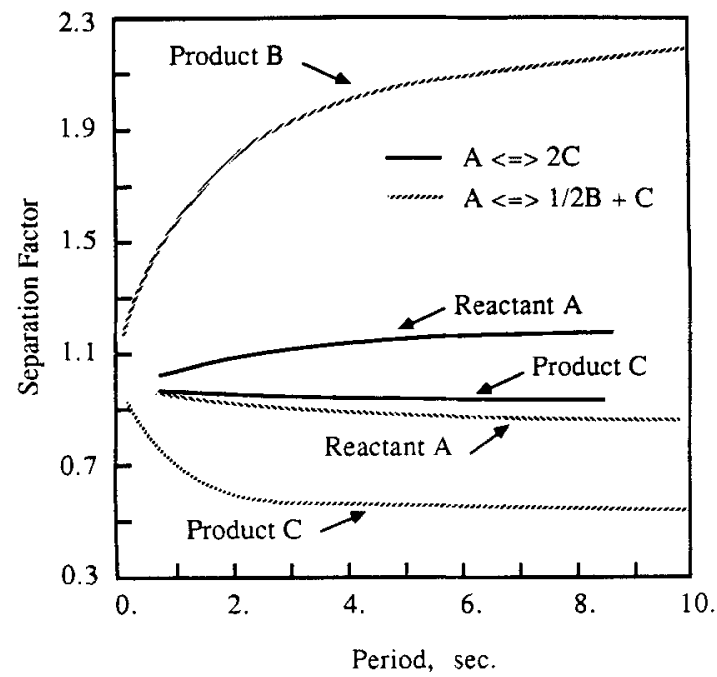

Figure 10. Period vs. separation factor. is true. Higher capacities occur at rapid cycling because most of the gas fed into the reactor does not travel far down the bed before being exhausted. The shapes of these curves were found to be insensitive to the delivery flow rate.

The effect of cycle period and reaction stoichiometry upon the separation performance of an EPSR is shown in Figure 10. Single-reactant and/or -product separations are poor because the component enrichment depends upon the average pressure difference between the delivery and exhaust streams. Separation between multiple-product compounds is of the same order as PSA alone. At rapid cycling frequencies the bed experiences a mean inlet feed pressure, and no separation occurs. At the other end of the period range the fraction of the feed recovered in the delivery stream increases to a level where product enrichments level off.

The effects of cycle feed fraction upon the dimensionless flow rates and separation are shown in Figures 11 and 12 . Similar to the cycle period, there exists a lower bound for the feed fraction at which point the delivery flow rate cannot be sustained. At a feed fraction of one the process operates as a plug flow reactor. As would be expected, the dimensionless flow rates go through a maximum, and the delivery cut goes through a minimum.

The effect of the cycling period upon conversion depends upon the sign of $\Delta v$. For a reaction that creates moles, the conversion decreases slightly with increasing feed fraction, while for a reactions which destroys moles the conversion increases slightly with increasing feed fraction. The pressure dependency of the reaction equilibrium expression is controlling and again provides the explanation. Most of the process gas leaves via the exhaust stream. The feed end of the PSR is at higher average pressure during the exhaust portion of the process cycle when the feed fraction is closer to one, thus the trends observed are expected. There is a local minimum in conversion at a low feed fraction for the reaction $2 A \rightarrow C$, since at low feed fractions the delivery stream becomes a significant fraction of the total gas exiting the reactor.

The most unusual behavior of the EPSR when varying the feed fraction concerns the separation peformance, Figure 12. A significant feature of these plots is the fact that component

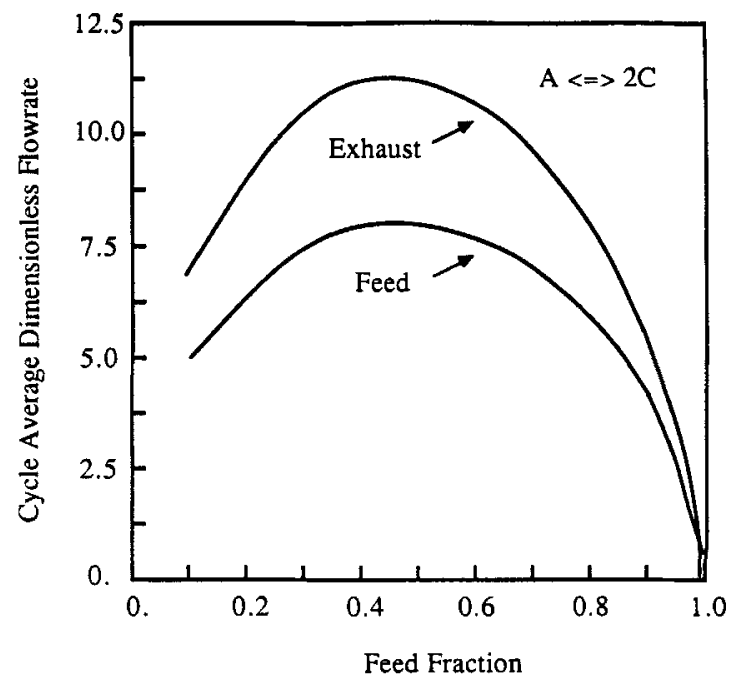

Figure 11. Feed fraction v8. cycle average dimensionless fiow rate. 


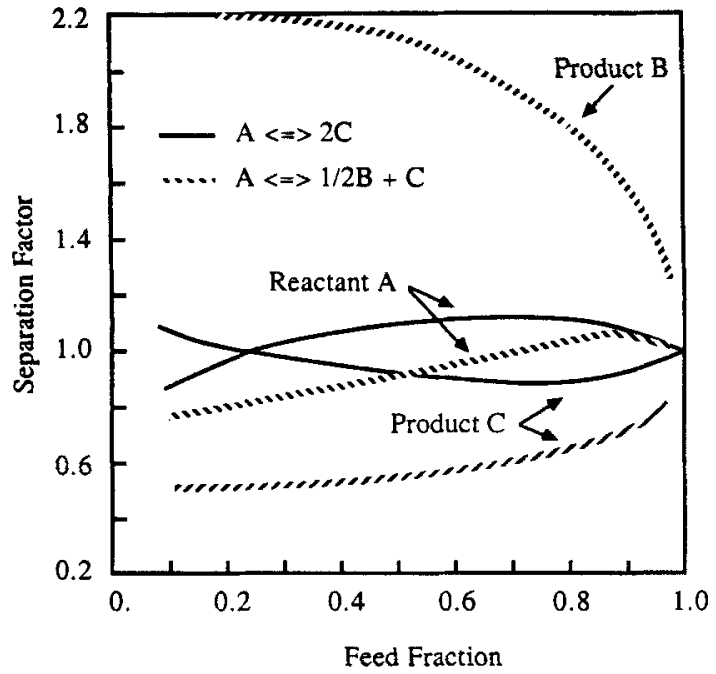

FIgure 12. Feed fraction v8. separation factor.

enrichments undergo reversal. The separation reversals are limited to those components that are single reactants or products. The pressure waves within the packed bed control the separation of these species. For the reaction $A-2 C$ at low feed fractions (above the minimum required for the set delivery stream flow rate) separation of the components occurs. As the feed fraction increases, separation factors approach one until a critical feed fraction where there is no separation. The separation factors then reverse and go through either a local maximum or minimum.

This effect is due to the difference between the average pressures at the feed end of the EPSR and delivery system. At low feed fractions, during the exhaust portion of the cycle, the average pressure at the feed end of the EPSR is lower than the average delivery pressure. This results in reactant enrichment in the exhaust stream for a positive $\Delta v$ and in the delivery stream for a negative $\Delta v$. As the feed fraction is increased a critical feed fraction is reached where both delivery and exhaust cycle average pressures are equal. Further increase in the feed fraction results in the delivery average pressure being greater than the average exhaust end pressure, thus the separation reversal.

For the reaction $A-1 / 2 B+C$ components $B$ and $C$ do not show separation reversals and exhibit enrichment at levels beyond that of single reactants or products. Separation reversal of the reactant is explained by the reversal of the pressure difference between the delivery and exhaust streams. The critical feed fraction varies with $\Delta v$ and the number of components present.

For both reaction cases the limit at a feed fraction of one is almost no separation. As the feed fraction approaches one, the pressure waves in the bed level out, until at the limit the bed is at a constant pressure profile. For the delivery flow rates employed here the pressure drop across the bed is almost zero. Due to this fact, material leaving the reactor at either end is basically at the same composition, that of equilibrium conversion at the constant bed pressure.

\section{Conclusions}

A new unit operation has been described, one that utilizes a rapid pressure swing cycle in a catalytic-adsorbent bed to effect both continuous pas-phase reaction and separation. For the case of extremely rapid reactions, a general multicomponent perturbed reaction-sorption equilibrium model has been formulated, and solved for two- and three-component reactions for various parameters and reaction stoichiometries.

System performance, in terms of capacity, separation, and extent of reaction are directly affected by various design and physical parameters. The capacity and separation performance for EPSR is of the same order of magnitude as equilibrium PSA alone. For single-reactant or single-product systems the component enriched in a particular EPSR exit stream has been found to depend upon both the reaction stoichiometry and feed fraction of the process cycle. Adsorption selectivities affect EPSR capacity, as do other PSA parameters and the reaction $K_{\text {eq }}$, but adsorption selectivities do not change component enrichments for single-reactant or -product systems. The common underlying cause of these separation reversals is the pressure dependency of the reaction equilibrium expression. For reaction systems with multiple products or reactants, separations are greater than single-component cases, and are affected by adsorbent properties in the same manner as in PSA. Additional degrees of freedom in the reaction equilibrium constraint for multiple reactants or products explains the ability to separate components in the latter case.

Dimensional analysis yields the interactions between several variables. The permeability and gas viscosity directly affect the capacity of the system but not the relationship between the fraction of feed gas recovered in the delivery stream, the separation, and the extent of reaction achieved. The perturbed reactionsorption model also predicts that a shorter periodic reactor can process more reactants without a loss of conversion or separation. Conversions are not enhanced, but separations are allowed.

In most unit operations an equilibrium theory results in a model that predicts capacities, conversions, or separations that are optimistic, thus representing a best limiting case. For periodic separating reactors the perturbed reaction-sorption equilibrium theory represents a limiting case that is conservative in its predictive nature. Slow reaction rates would predict greater conversions and separations than the perturbed equilibrium model. This is the situation for the chromatographic reactor studied by Chu and Tsang (1971). Consider for example the rate-limited reaction $A-1 / 2 B+C$. Without the instantaneous conversion of the feed, and the constraint that the reactant always be at reaction equilibrium with the products, increased separation of component $A$ down the length of the PSR would be possible. PSR performance would change, as the increased separation between components $B$ and $C$ would reduce the rate of back reaction, thereby increasing the total conversion of $A$. Furthermore, conversion now also depends upon the residence time of the reactant within the PSR. Depending upon the adsorption characteristics of the packing and the flow pattern (as determined by the total cycle time, feed fraction delay fraction, etc.) a PSR could be designed so that the reactant has a longer residence time within the bed than the product(s), and thus limit the reverse reaction.

In short, periodic separating reactors have two limits, that of pure PSA at one end where the separation performance of the system controls, and a perturbed reaction equilibrium as the other limit where it has been determined the reaction phenomenon controls. A PSR system with finite rates of reaction will likely show maxima and minima between these limits. 


\section{Acknowledgment}

The authors gratefully acknowledge the aid of Raymond Wright in conducting part of the simulations.

\section{Notation}

$A$ - cross-sectional area of periodic separating reactor, $\mathrm{cm}^{2}$

$f=$ fraction of process period

$J$ - rank of atom matrix for reaction under consideration

$K_{e q}$ - reaction equilibrium constant, atm ${ }^{\Delta \nu}$

$\vec{k}$ = permeability of packed bed, $\mathrm{g} \cdot \mathrm{cm} / \mathrm{atm} \cdot \mathrm{s}^{2}$

$L$ - length of packed bed, $\mathrm{cm}$

$\boldsymbol{M}=$ molecular weight of a component, $\mathrm{mg} / \mathrm{mg} \mathrm{mol}$

$N$ - total number of components in system

$O$ - cumulative molar output from single tank during depressurization, $\mathrm{mg} \cdot \mathrm{mol}$

$P$ - pressure, atm

$Q$ - cycle average molar flow rate, $\mathrm{mg} \mathrm{mol} / \mathrm{s}$

$R$ - ideal gas constant, atm $\cdot \mathrm{cm}^{3} / \mathrm{mg} \mathrm{mol} \cdot \mathrm{K}$

$T$ - absolute temperature, $\mathrm{K}$

$t=$ time into cycle, $s$

$V$ - volume, $\mathrm{cm}^{3}$

$x$ - distance down packed column from feed point, $\mathrm{cm}$

$y=$ mole fraction in vapor phase

$Z$ = pressure, $P / P_{f}$

\section{Greek letters}

$\alpha=$ constant in linear adsorption isotherm, $\mathrm{mg} / \mathrm{mol} / \mathrm{atm} \cdot \mathrm{g}$ ads

$\gamma$ - bed capacity, Eq. 2

$\Delta v=$ net change in total moles due to reaction

$\epsilon=$ porosity of packed bed

$\eta$ - number of atoms of a certain type in subscripted chemical component

$\theta=$ time, $t / \tau$

$\lambda=$ length down packed bed, $x / L$

$\mu$ - average viscosity of gases, $\mathrm{g} / \mathrm{cm} \cdot \mathrm{s}$

$\rho=$ density of adsorbent or adsorbent $/$ catalyst, $\mathrm{g} / \mathrm{cm}^{3}$

$v$ - stoichiometric coefficient of subscripted chemical component

$\tau$ - cycle period, $s$

\section{Subscripts}

$D$ - delay portion of cycle

$d$ - delivery stream

$e$ - exhaust stream or part of cycle

$f$ - feed stream or part of cycle

$i=i$ th chemical component of $N$ present

$j=j$ th type of atom of total present

min - minimum pressure, $P_{s} / P_{f}$

$R$ - reflux void volume

\section{Literature cited}

Adam, Y., "Nonlinear Instability in Advection-Diffusion Numerical Models," Appl. Math. Modeling, 9, 434 (1985).

Amariglio, H., and G. Rambeau, "On the Possibility of Rate Improvements by a Periodic Operation of Catalytic Reactors," Chem. Eng. Sci., 39, 1433 (1984).

Bailey, J. E., "Periodic Operation of Chemical Reactors: A Review," Chem. Eng. Commun., 1, 111 (1973).

Cannon, M. R., "A New Type of Distillation and Absorption Column." Oil Gas J., 51, 268 (July 28, 1952).

Chu, C., and L. C. Tsang, "Behavior of a Chromatographic Reactor," Ind. Eng. Chem. Process Des. Dev., 10(1), 47 (1971).

Cutlip, M. B., "Concentration Forcing of Catalytic Surface Rate Processes," AIChE J., 25, 502 (1979).

Feimer, J. L., A. K. Jain, R. R. Hudgins, and P. L. Silveston, "Modeling Forced Periodic Operation of Catalytic Reactors," Chem. Eng. Sci., 37, 1797 (1982).

Imbihl, R., M. P. Cox, G. Ertl, H. Muller, and W. Brenig, "Kinetic Oscillations in Catalytic CO Oxjdation on Pt(100): Theory," $J$. Chem. Phys., 83, 1578 (1985).

Jain, A. K., R. R. Hudgins, and P. L. Silveston, "A Model to Unni's Experiments on Cyclic Catalytic Oxidation of $\mathrm{SO}_{2}$ " Chem. Eng. Sci., 36, 231 (1981).

- "Adsorption/Desorption Models: How Useful for Predicting Reaction Rates Under Cyclic Operation?" Can. J. Chem. Eng., 61, 46 (1983).

Kevrekidis, I. G., L. D. Schmidt, and R. Aris, "Some Common Features of Periodically Forced Reacting Systems," Chem. Eng. Sci., 41, 1263 (1986).

Kowler, D. E., and R. H. Kadlec, "The Optimal Control of a Periodic Adsorber. I: Experiment; II: Theory," AIChE J., 18(6), 1207 (1972).

Lynch, D. T., "On the Use of Adsorption/Desorption Models to Describe the Forced Periodic Operation of Catalytic Reactors," Chem. Eng. Sci., 39, 1325 (1984).

Lynch, D. T., G., Emig, and S. E. Wanke, "Oscillations During CO Oxidation over Supported Metal Catalysts. III: Mathematical Modeling of the Observed Phenomena," J. Catal., 97, 456 (1986).

Mihail, R., and R. Paul, "A Model to Unni's Experiments on Cyclic Catalytic Oxidation of SO," Chem. Eng. Sci., 34, 1058 (1979).

Salmi, T., L. E. Lindfors, and S. Bostrom, "Modeling of the High-Temperature Water-Gas Shift Reaction with Stationary and Transient Experiments," Chem. Eng. Sci., 41, 929 (1986).

Sincic, D., and J. E. Bailey, "Pathological Dynamic Behavior of Forced Periodic Chemical Processes," Chem. Eng. Sci., 32, 281 (1977).

Turnock, P. H., and R. H. Kadlec, "Separation of Nitrogen and Methane via Periodic Adsorption," AIChE J., 17, 335 (1971).

Williams, E. D., W. H. Weinberg, and A. C. Sobrero, "CO on Ru(001): Island Size and Disordering," J. Chem. Phys., 76, 1150 (1982).

Manuscript received Oct. 22, 1986, and revision received Feb. 17, 1987. 